\title{
UM ALGORITMO PARA CONTORNO AUTOMÁTICO DE DADOS GEOLÓGICOS
}

\author{
J.K. Yamamoto'
}

\section{RESUMO}

Este trabalho apresenta um algorftmo para contorno automático de dados geológicos, bem como de quaisquer outros referenciados geograficamente. Esse algoritmo foi baseado num outro escrito em linguagem Basic, reescrito em linguagem Turbo Pascal e adaptado para permitir a plotagem contínua das curvas de isovalores. É também apresentada uma revisão dos métodos correntes para contorno automático de malhas regulares e uma descrição dos algoritmos para armazenamento, classificação e rastreamento das intersecções das curvas, os quais permitem a plotagem contínua das mesmas. Esses algorttmos, com algumas modificações, poderiam ser utilizados para se fazer o reconhecimento automático de padrões em mapas, tais como contatos geológicos e redes de drenagens.

\section{ABSTRACT}

It is presented an algorithm for automatic contouring of geological data and geographical reference data. This algorithm was derived from on already published in Basic language, rewritten in TURBO PASCAL language, and adapted to perform continuous plotting of contour curves. This paper presents a revision of current methods for automatic contouring of regular grids and describes the algorithms for storing, sorting, and tracking the intersection segments which allow the continuous plotting. These algorithms, with some modifications, could be used to perform automatic

\footnotetext{
'Departamento de Geologia Econômica e Geofísica Aplicada, Instituto de Geociências/USP.
} 
pattern recognition in maps (for example, geological boundaries and drainage networks).

\section{INTRODUÇÃO}

Os levantamentos geológicos de uma determinada região levam, quase que invariavelmente, à coleta de dados distribuidos em área. Esses dados, em função do interesse e da natureza da investigação, podem ser: estruturais, sedimentológicos, geoquímicos, geofísicos, etc. Assim, a interpretação geológica dos resultados de análises e/ou medidas é feita geralmente sobre mapas de contorno de isovalores, que são a forma mais comumente utilizada pelo geólogo para representação gráfica de dados distribuidos em área.

A geração dos mapas de contorno de isovalores é bastante trabalhosa, principalmente quando o número de pontos é multo grande e estes são irregularmente distribuídos. É importante ressaltar que, ainda hoje, grande parte dos mapas publicados em trabalhos cientfficos são ainda traçados manualmente, devido, principalmente, ao não conhecimento das técnicas de contorno automático que podem ser utilizadas, bem como suas vantagens e limitações. A principal vantagem em se produzir um mapa de contorno de forma manual é a sensibilidade que o pesquisador introduz por conhecer o dado e o tipo de variação mais provável; entretanto, isto torna o produto um tanto subjetivo, uma vez que diferentes pesquisadores produzirão diferentes mapas.

Este trabalho apresenta uma descrição sucinta dos métodos de contorno automático (células e rastreamento) existentes e apresenta um algorttmo que, adaptado ao método das células, permite obter o traçado contínuo das curvas de isovalores.

\section{CONTORNO AUTOMÁTICO DE MALHAS REGULARES}

O contorno automático de dados geológicos ou quaisquer outros referenciados geograficamente, é obtido geralmente a partir de malhas regulares em cujos nós são conhecidos os valores da variável de interesse. As referências consultadas (PETTINATI, 1983; OLEA, 1975; entre outras) mostram que o desenho automático de malhas regulares é o mais indicado para obtenção de um produto final melhor em termos estéticos, bem como pelas facilidades computacionais advindas da sua utilização. As malhas regulares podem tanto resultar do planejamento da amostragem, como também através da interpolação dos nós de um arranjo regular, quando os dados se encontrarem 
irregularmente distribuídos.

Assim, dada a malha regular, pode-se fazer o seu contorno automático, basicamente por meio de dois métodos: rastreamento e células.

O primeiro deles, denominado método do rastreamento, segue as curvas de contorno, curva por curva, a partir de algum ponto inicial, de onde são pesquisados os demais pontos que pertencem a uma determinada curva até coincidir ou com o ponto inicial (curva fechada) ou com a borda da malha regular (curva aberta). Este método é geralmente utilizado nos pacotes gráficos para contorno automático, disponiveis comercialmente.

O último, denominado método das células, pesquisa célula por célula, segundo alguma sequiência preestabelecida, até cobrir toda a malha. Em cada célula o algoritmo determina todas as intersecções de todas as curvas de contorno que por ventura estejam presentes, unindo-as com segmentos de retas. Exatamente sobre esse último método foi apresentada por BOURKE (1987) uma sub-rotina em linguagem BASIC. O programa de Bourke faz o contorno automático pelo método das células, porém subdividindo-as em quatro triângulos, que são pesquisados individualmente quanto às possiveis intersecções. Essa subdivisão do interior das células em triângulos, torna as curvas mais suaves que aquelas traçadas diretamente das células, como foi demonstrado por BATCHA \& REESE (1964). Esse método, contudo, não permite fazer anotações do valor da curva, bem como aplicar métodos para suavização das mesmas (vide, por exemplo, CLNE, 1974) se estas apresentarem-se angulosas, pois as curvas, na realidade, são segmentadas e não contínuas. Se este método for utilizado para saída em "plotter", af surgirá o maior problema que é justamente o grande tempo de plotagem requerido para o desenho das curvas de contorno, tendo em vista a necessidade de deslocamento da pena do "plotter" a cada segmento de reta.

Assim, foi desenvolvido um algoritmo que, a partir das intersecções determinadas pelo método das células, permite fazer o armazenamento temporário de todas as intersecções de uma determinada curva, classificá-las de tal modo que as curvas sejam contínuas e, finalmente, fazer o traçado no monitor ou no plotter. Apresenta-se neste trabalho o programa CONREC.PAS reescrito em linguagem Pascal a partir do programa CONREC.BAS de BOURKE (1987), juntamente com dois novos procedimentos adicionais que permitem, respectivamente, fazer o armazenamento temporário de todos os segmentos de uma determinada curva e fazer a classificação desses para o traçado contínuo da mesma. Para fins do presente trabalho o programa original foi modificado para pesquisar curva por curva em todas as células da malha regular, ao invés do original que pesquisa todas as curvas, célula por célula até cobrir toda a malha regular. 


\section{O ALGORITMO}

Dentro do próprio procedimento CONREC, foram colocados os dois novos procedimentos que se chamam: ARMAZENA e RASTREIA. O primeiro deles armazena todas as intersecções de uma determinada curva enquanto a mesma estiver sendo processada. $O$ procedimento RASTREIA recebe como entrada todas as intersecções (pares de pontos) e faz várias classificações, de tal maneira que todos os pares de pontos fiquem ligados entre si, formando uma seqũência contínua de pontos que definem uma determinada curva de contorno.

\section{PROCEDIMENTO ARMAZENA}

Este procedimento é chamado todas as vezes que forem encontradas intersecções entre o plano da curva de contorno e os triângulos internos das células. O procedimento recebe como argumentos de entrada dois pares de pontos ( $x 1 r, y 1 r)$ e (x2r, y2r), que são transformados para o campo inteiro (x1i, y1i) e (x2i,y2i). Foi adotada esta transformação para economizar memória e, principalmente, para tornar mais rápida a operaçăo de classificação. 0 campo dos números inteiros em TURBO PASCAL permite trabalhar com números dentro do intervalo -32768 a 32767 , portanto suficientes para representação com bastante precisão das coordenadas de mapas. Foram utilizadas também variáveis tipo ponteiro para permitir o processamento de mapas que requeiram mais que 64 Kbytes de memória. Os pontos recebidos na entrada do procedimento são testados e armazenados de tal modo que a coordenada " $x$ " do primeiro par seja menor que a mesma do segundo; caso essas duas coordenadas sejam iguais, os pontos são ordenados para que a coordenada " $y$ " do primeiro par seja menor que a mesma do segundo. Assim, todas intersecções encontradas em uma determinada curva são armazenadas, quando então é chamado o procedimento RASTREIA.

\section{PROCEDIMENTO RASTREIA}

Este procedimento recebe como entrada todas as intersecções pertencentes a uma determinada curva, armazenadas em quatro vetores: $x 1, y 1, x 2$ e y2, sendo os dois primeiros para armazenar as coordenadas iniciais da intersecção e os outros dois para as coordenadas finais da intersecção. 
Todos os pontos são então classificados em ordem crescente de x1. Nos pontos ainda é verificado se a coordenada $x 1$ de um determinado ponto é igual ao do ponto anterior; se forem iguais, é feita uma troca entre as coordenadas $\times 1$ e $\times 2$ do ponto anterior. Em seguida, é feita uma varredura em todos os valores armazenados, verificando-se se a coordenada $x 1$ de um ponto é igual a coordenada $\times 2$ do ponto anterior; se forem iguais, armazena-se somente o primeiro ponto com o código 2, caso contrário armazena-se o primeiro ponto com o código 2 e o segundo ponto com o código 3. Fol utilizada a convenção padrão da indústria para identificar os comandos de movimentação de pena do "plotter", ou seja, o código 3 comanda o "plotter" para ir até a coordenada $(x, y)$ especificada com a pena levantada, enquanto o código 2 é utilizado para comandar o "plotter" para ir até o ponto especificado com a pena abaixada. Após estas operações são identificados todos os segmentos que compõem uma determinada curva de contorno. Cada segmento começa com o código 3, seguido por uma série de pontos com o código 2 . O problema agora é unir todos os segmentos que compõem uma determinada curva de contorno, ou trecho dela. Para isso, foi utilizado um algorftmo baseado na classificação bidimensional, desenvolvido por YAMAMOTO (1986) para fazer a busca dos pontos vizinhos mais próximos na interpolação de malhas regulares por métodos locais, dos segmentos de curva, a partir da discretização da área do mapa em células, cujas dimensões podem ser variáveis e até iguais às dimensões da malha regular original. Entretanto, o número de células da malha original pode ser excessivo, aumentando desnecessariamente o tempo de processamento. Para fins de ilustração utilizou-se para o programa CONREC.PAS, uma subdivisão da área do mapa em 100 células, sendo 10 na direção do eixo $X$ e outras 10 na direção do eixo Y. Cada segmento de curva, constituido por uma série de pontos, pode ser identificado em termos do ponto inicial (ponto de código 3) e do ponto final (ponto de código 2). Aos pontos inicial e final do segmento de curva pode-se atribuir os respectivos números das células, às quais eles pertencem. A numeração das células é feita seqüencialmente a partir do canto inferior esquerdo: de baixo para cima e da esquerda para a direita. O número de qualquer célula, localizada pela linha e coluna a que pertence, pode ser encontrado pela relação a seguir:

$$
\text { ncel }=1 i n+\text { nrow } *(\operatorname{col}-1)
$$

onde: ncel é o número da célula;

lin é a linha a que pertence a célula;

col é a coluna a que pertence a célula;

nrow é o número de linhas, ou o número de células na direção do eixo Y. 
Assim, para cada segmento de curva tem-se o número da célula do ponto inicial e o número da célula do ponto final, que são determinados através da relação (1). As variáveis lin e col são determinadas a partir das coordenadas dos pontos, como segue:

$$
\begin{aligned}
& \operatorname{lin}=(y \operatorname{div} d y)+1 \\
& \operatorname{col}=(x \operatorname{div} d x)+1
\end{aligned}
$$

onde: y é a coordenada y do ponto;

dy é a abertura da célula na direção y;

$x$ é a coordenada $x$ do ponto;

dx é a abertura da célula na direção $x$;

div é o operador Pascal que indica a divisão entre inteiros.

Em seguida, os pontos iniciais e os pontos finais de todos os segmentos de curva são classificados em ordem crescente do número de células. Após essas classificações tem-se duas listas ordenadas, uma com todos os pontos iniciais $\theta$ outra com todos os pontos finais dos segmentos de curva. A partir desse momento começa a operação de rastreamento propriamente dita, conforme descrito a seguir.

Retira-se da lista o primeiro segmento, cujas coordenadas iniciais são armazenadas

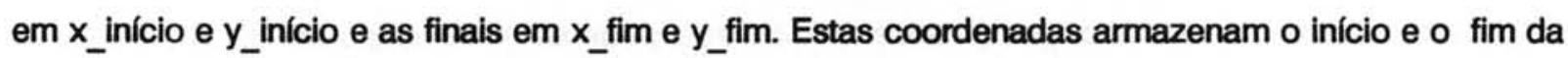
curva rastreada. Pelas coordenadas $x_{-}$início e y_início, localiza-se a célula na qual se encontra a extremidade da curva rastreada. Nesta célula é verificado se existe algum segmento que tenha uma de suas extremidades coincidindo com as coordenadas $x_{-}$início e y_início da curva rastreada; se existir 0 segmento é ligado à curva rastreada e retirado da lista ordenada. As coordenadas $x_{-}$início e y_início da curva rastreada são atualizadas com os valores das coordenadas da extremidade do segmento que foi ligado à mesma. Dessa forma o processamento continua até que não se encontre nenhum segmento na célula pesquisada.

O procedimento descrito é repetido com outra extremidade da curva rastreada, ou seja, com as coordenadas $x_{\text {f fim e }} \mathrm{y}_{-}$fim. Da mesma forma, verifica-se na célula a qual pertencem as coordenadas $x_{-}$fim e y_fim, da curva rastreada, a existência de algum segmento, que tenha uma de suas extremidades coincidindo com aquela da curva rastreada; se existir o segmento é adicionado à

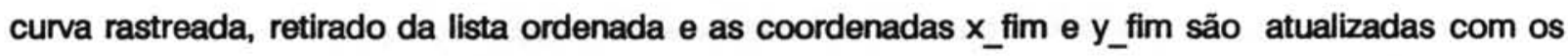


novos valores.

No final do processamento, quando o último segmento é adicionado, pode-se ter ou as coordenadas $x_{-}$início e y_início coincidindo com as coordenadas $x_{-}$fim e y_fim (curva fechada) ou interceptando as bordas do mapa (curva aberta).

Os algoritmos apresentados nos procedimentos ARMAZENA E RASTREIA poderiam, com pequenas modificações, ser utilizados para se fazer o reconhecimento automático de padrões a partir de dados introduzidos via mesa digitalizadora. Como exemplos podem ser citados o reconhecimento de contatos em mapas geológicos e o reconhecimento de redes de drenagem.

\section{EXEMPLO DE APLCAÇÃO}

Como exemplo de aplicação, escolheu-se três conjuntos de dados de elevações de terrenos, lidos sobre cartas topográficas 1:50 000 do Instituto Brasileiro de Geografia e EstatísticaIBGE, quais sejam: Cunha, Campos do Jordão e Botucatu, todas no Estado de São Paulo.

Os dados foram lidos sobre uma malha regular de 15 por 15 nós, totalizando 225 pontos de dados por conjunto.

$O$ arquivo tipo texto DADOS.REG, que acompanha a listagem do programa CONREC.PAS, contém os três conjuntos de dados introduzidos seqüencialmente do canto inferior esquerdo da malha regular, para cima e da esquerda para a direita. Em cada linha encontram-se os dados de topografia das áreas de Cunha, Campos do Jordão e Botucatu, respectivamente, introduzidos conforme a ordem descrita acima.

Para utilizar o programa CONREC.PAS, procede-se da seguinte forma:

a) carregar o compilador TURBO PASCAL, colocando o disquete com o mesmo no drive ativo e teclando TURBO;

b) teclar $\mathrm{Y}$, em resposta a: Include error messages?

c) teclar W (Work File Name) e fornecer o nome do programa: CONREC;

d) teclar R, para compilar o programa para a memória e executar;

e) em resposta ao programa, fomecer as seguintes informações (para processar a área de Cunha):

qual o nome do arquivo da malha regular = DADOS.REG 
qual a opção de leitura $=1$

qual o numero de nós na direção $x=15$

qual o numero de nós na direção $y=15$

curva minima $=\mathbf{2 0 . 0 0}$ quer modificar $(s / n)=S$

qual o valor da curva mínima $=0$

curva máxima $=1660.00$ quer modificar $(s / n)=S$

qual o valor da curva máxima $=1600$

eqüidistância $=160.00$ quer modificar $(s / n)=S$

qual o valor da eqüidistância $=100$

f) o mapa de contorno das curvas de isovalores será desenhado no monitor no modo CGA (Color Graphics Adapter) de 320 por 200 pontos;

g) para imprimir o mapa use o programa do sistema operacional GRAPHICS, que permite copiar o mapa do monitor diretamente para a impressora matricial de pontos, conforme mostrado na Figura 1;

h) para terminar o processamento, aperte qualquer tecla, quando o monitor será limpo e o tempo de processamento (em segundos) será indicado.

i) para processar um novo conjunto voltar ao passo (d).

O resultado desse processamento é mostrado na Figura 1, onde tem-se representada a topografia da região de Cunha de 0 a $1600 \mathrm{~m}$, com eqüidistância de $100 \mathrm{~m}$.

Repetindo-se o processamento com opção de leitura igual a 2 e estabelecendo-se curva mínima igual a $900 \mathrm{~m}$, curva máxima igual a $1900 \mathrm{~m}$ e eqüidistância igual a $100 \mathrm{~m}$ tem-se o mapa de isovalores de elevações do terreno mostrado no desenho da Figura 2.

Finalmente, para opção de leitura igual a 3 e curvas mínima e máxima, respectivamente iguais a 450 e $675 \mathrm{~m}$, e intervalos de contorno de $25 \mathrm{~m}$, obtém-se no monitor o desenho mostrado na Figura 3.

\section{CONSIDERAÇÕES FINAIS}

É apresentado um programa de computação escrito em linguagem TURBO PASCAL, para microcomputadores nacionais de 16 bits do tipo PC, para se fazer o contorno automático de malhas regulares pelo método das células, com modificações que permitem obter o 


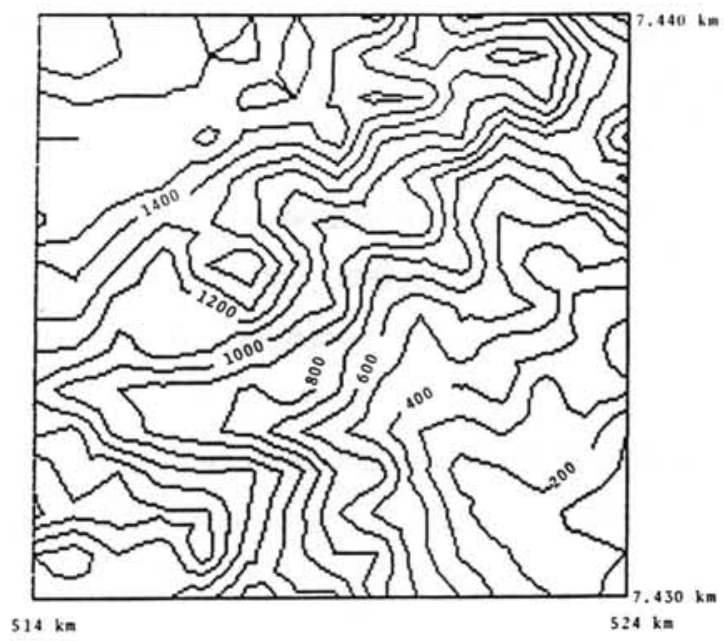

Figura 2 - Mapa de contorno de isovalores obtido para a área de Campos do Jordão, onde as curvas apresentam variaçăo entre 900 e $1900 \mathrm{~m}$ em intervalos de $100 \mathrm{~m}$. Algumas curvas foram identificadas manualmente para orientaçăo.

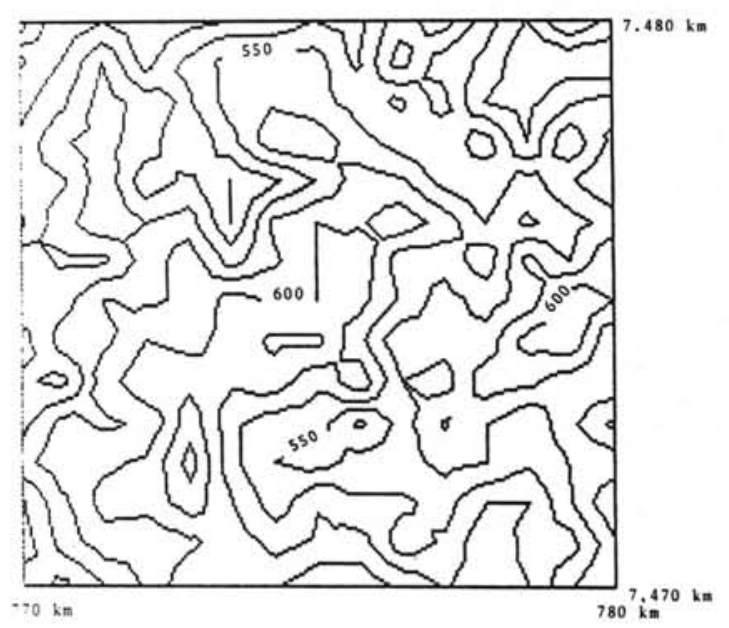

Figura 1 - Desenho reproduzido do monitor de vídeo, modo CGA da baixa resoluçăo, mostrando o mapa de contorno de isovalores obtido do processamento do programa CONREC.PAS para a área de Cunha. As curvas apresentam variaçăo entre 100 e $1600 \mathrm{~m}$ em eqüidistancia de $100 \mathrm{~m}$. Agumas curvas foram identificadas manualmente para orientaçăo.

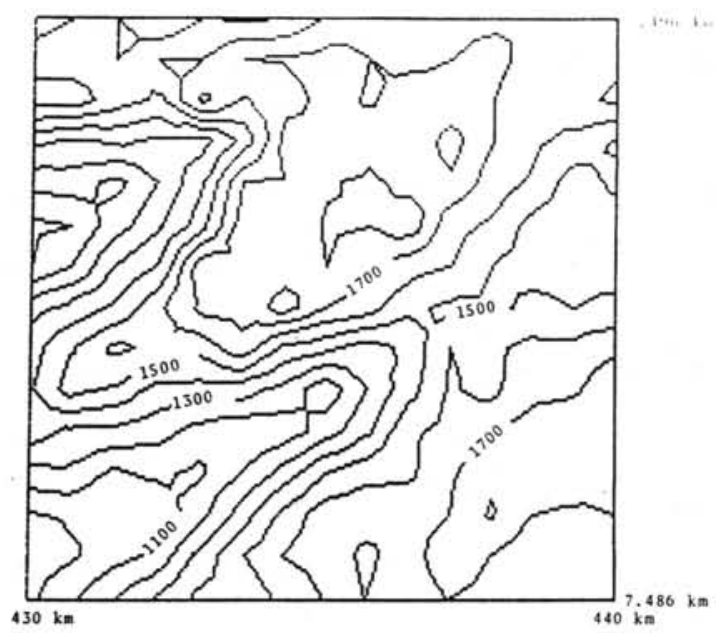

Figura 3 - Mapa de contorno de isovalores obtido com a opção de leitura três (área de Botucatu), representando variação na topografia de 450 e $675 \mathrm{~m}$ em intervalos de $25 \mathrm{~m}$. Algumas curvas foram identificadas manualmente para orientação. 
traçado contínuo das curvas de isovalores. As vantagens desta implementação são a possibilidade de suavização das curvas de isovalores através da aplicação de "splines" tensionados e a anotação do valor da curva em algum trecho da mesma.

Os procedimentos de armazenamento, classificação e rastreamento das curvas de contorno apresentados neste trabalho poderão, com algumas modificações, ser utilizados para reconhecimento automático de padrões em mapas, tais como: contatos geológicos e redes de drenagem.

Por fim, a publicação do algoritmo apresentado neste trabalho permitirá uma maior divulgação da técnica de contorno automático, incrementando a automatização de procedimentos até há pouco essencialmente manuais.

\section{REFERÊNCIAS BIBLIOGRÁFICAS}

BATCHA, J.P. \& REESE, J.R. (1964) Surface determination and automatic contouring for mineral exploration, extraction, and processing. Quaterly Colorado School of Mines, 59(1):1-14.

BOURKE, P.D. (1987) A contouring subroutine. BYTE, 12(6):143-150.

CLNE, A.K. (1974) Scalar and planar valued curved fitting using splines undertension. Communications of the ACM, 17(4):218-223.

OLEA, R.A. (1975) Optimum mapping techniques using regionalized variable theory. Kansas, Kansas Geological Survey. 137p. (Series on Spatial Analysis, 2)

PEITINATI, F. (1983) Modelamento digital e representação gráfica de superfícies. São Paulo, 177p. (Dissertação de Mestrado - Escola Politénica/USP).

YAMAMOTO, J.K. (1986) Representações gráficas espaciais em geologia: aplicações no complexo alcalino de Anitápolis. São Paulo, 167p. (Dissertação de Mestrado - Instituto de Geociências/USP). 